\title{
REVIEW ARTICLE \\ What is the evidence for giving chemoprophylaxis to children or students attending the same preschool, school or college as a case of meningococcal disease?
}

\author{
W. HELLENBRAND ${ }^{1 *}$, G. HANQUET ${ }^{2}$, S. HEUBERGER ${ }^{3}$, S. NIELSEN ${ }^{1}$, \\ P.STEFANOFF ${ }^{4}$ AND J. M. STUART ${ }^{5}$ \\ ${ }^{1}$ Immunization Unit, Robert Koch Institute, Berlin, Germany \\ ${ }^{2}$ Consultant epidemiologist (independent), Brussels, Belgium \\ ${ }^{3}$ Meningococcal Reference Laboratory, Austrian Agency for Food and Health Safety Graz, Austria \\ ${ }^{4}$ National Institute of Public Health, National Institute of Hygiene, Warsaw, Poland \\ ${ }^{5}$ Consultant epidemiologist (independent), Ausseing, France
}

(Accepted 5 July 2011; first published online 11 August 2011)

\section{SUMMARY}

We performed a systematic literature review to assess the effectiveness of chemoprophylaxis for contacts of sporadic cases of invasive meningococcal disease (IMD) in educational settings. No studies directly compared IMD risk in contacts with/without chemoprophylaxis. However, compared to the background incidence, an elevated IMD risk was identified in settings without a general recommendation for chemoprophylaxis in pre-schools [pooled risk difference (RD)

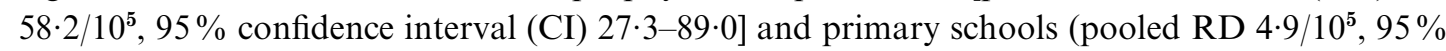

CI 2.9-6.9) in the $\sim 30$ days after contact with a sporadic IMD case, but not in other educational settings. Thus, limited but consistent evidence suggests the risk of IMD in pre-school contacts of sporadic IMD cases is significantly increased above the background risk, but lower than in household contacts (pooled RD for household contacts with no chemoprophylaxis vs. background incidence: $480 \cdot 1 / 10^{5}, 95 \%$ CI 321·5-639.9). We recommend chemoprophylaxis for pre-school contacts depending on an assessment of duration and closeness of contact.

Key words: Chemoprophylaxis, educational settings, meningococcal disease, public health.

\section{INTRODUCTION}

Invasive meningococcal disease (IMD) in Europe for the most part occurs as sporadic cases, with most transmission leading to asymptomatic carriage [1]. In several European studies investigating the proportion of subsequent, epidemiologically linked cases, these comprised $3-16 \%$ of all cases [2-6], with higher proportions reported in higher incidence settings $[3,5,6]$. For instance, in Belgium the proportion of secondary

\footnotetext{
* Author for correspondence: Dr W. Hellenbrand, Robert Koch Institute, DGZ-Ring 1, 13086 Berlin, Germany.

(Email: hellenbrandw@rki.de)
}

cases (including co-primary cases) was $5 \cdot 2 \%$ in 1971-1973 and $2 \%$ in 1974-1976, with IMD incidence decreasing from $\sim 5$ to 1 IMD cases $/ 100000$ inhabitants during 1971-1976 [3]. In Frediksborg county, Denmark, the proportion of secondary cases was $5-16 \%$ from 1987 to 1989 , during which the overall incidence was extremely high at between $9 \cdot 7$ and $14 \cdot 1[6]$. While observational studies have shown that chemoprophylaxis of household contacts of persons with IMD to eradicate nasopharyngeal carriage of Neisseria meningitidis reduces the risk of subsequent cases in those contacts [7,8], this has not been shown for contacts in other settings, although there are numerous reports on the occurrence of 
secondary cases in pre-school [2-5, 9-17], school $[2-5,11,17-30]$, and university or college settings [2, 31-35]. Asymptomatic transmission of the index strain has also been shown in these settings, although to a lesser extent than in household settings $[10,16$, $36,37]$. A survey of 12 European countries performed in 2006 revealed variation in policy regarding chemoprophylaxis for contacts between countries as well as within countries over time [38]. This was corroborated by unpublished data from a recent survey [39], in which 12/28 European countries did not recommend chemoprophylaxis for pre-school/ day-care contacts (referred to as pre-school from hereon in) of a case of IMD while 16 did. Of these, three recommended chemoprophylaxis for the entire institution and 10 for contacts in the group or class of the index patient (M. Hoek, personal communication). The lack of a common approach to policy development in this area was in part attributed to uncertainty around the effectiveness of preventive measures [39]. Therefore, the European Centre for Disease Prevention and Control (ECDC) launched the development of guidance on the public health management of sporadic cases of meningococcal disease. This involved systematic literature reviews and the application of GRADE methodology [40-43] to grade the evidence and strength of recommendations [44]. In this paper, we present the results of a systematic literature search performed as part of this project to search for direct and indirect evidence on the effectiveness of chemoprophylaxis in educational settings.

\section{METHODS}

We performed a systematic literature review looking for direct and indirect evidence for the effectiveness of chemoprophylaxis in pre-school, school and college contacts of primary IMD cases. As a similar search in 2004 had not found any direct evidence [7], we presumed the focus would lie on indirect evidence. Therefore, we specifically searched for data permitting a comparison of the incidence of IMD in contacts of primary cases in educational settings with the background risk of sporadic cases. To enable an explicit comparison with risk in household settings, for which there is direct evidence for the effectiveness of chemoprophylaxis of close contacts [7], we also searched for analogous data for household contacts of sporadic IMD cases.

\section{Search strategy}

We searched the literature up to December 2009 in Medline (from 1960), EMBASE (from 1974), Global health (from 1972), Cochrane database of systematic reviews and the Cochrane central register of controlled trials through the German Institute of Medical Documentation and Information (DIMDI, http:// www.dimdi.de/static/en/index.html). The following search string was used to retrieve relevant papers: (meningoc? OR neisseria meningit?) AND (chemoprev? OR ?prophyla? OR antibiotic?) AND (transmission OR contact? OR second? OR attack OR cluster? OR outbreak?) AND (?school? OR day care OR nurser? OR child care OR college? OR universit? OR dormitor?), yielding 310 abstracts. To search for further, indirect evidence as described above, we dropped the term (chemoprev? OR ?prophyla? OR antibiotic?) and added the term AND (incidence or risk) to identify any studies investigating subsequent cases of meningococcal disease in contacts of primary cases that would also allow comparison with the background incidence of IMD. This yielded 386 additional papers.

The following search string was used to identify studies comparing the incidence of subsequent cases in contacts in household settings with background incidence: (meningoc? OR neisseria meningit?) AND (transmission OR contact? OR second? OR attack OR cluster? OR outbreak?) AND (household or family). This yielded an additional 238 papers, for a total of 934 retrieved references that were screened.

\section{Inclusion and exclusion criteria}

Papers in any European language were accepted. We individually assessed abstracts for relevance to the question and reviewed full papers on relevant abstracts. Papers were selected for inclusion in the evidence assessment if they described analytical or observational studies with comparison groups or studies permitting comparison of incidence in contacts of at least 10 cases in the defined settings with the background incidence of sporadic IMD cases. We examined reference lists in pertinent papers for other relevant publications, and searched Google Scholar for citations of identified key papers.

\section{Identification of unpublished data}

To minimize bias, epidemiologists and microbiologists with expertise in meningococcal disease 
across Europe were identified through established Meningococcal Networks (Meningococcal Disease Society, Invasive Bacterial Infections Network based at ECDC) and asked for unpublished data that fitted our criteria. However, no further studies were identified.

\section{Comparison of IMD risk in contacts of primary cases with background incidence of sporadic cases}

In identified observational studies that provided information on subsequent cases of IMD in contacts of index cases of IMD in pre-school, school, college or household settings (see Appendix Table 1, available online) we extracted or calculated the incidence of subsequent cases in 1-30 days (or a period as close to this as possible) after contact to the index case (subsequent attack rate, SAR) in the retrieved studies for household and educational settings. This was then compared to the background age-specific incidence of sporadic cases (ISC) in the same time interval by calculating the relative risk (RR) and risk difference (RD). The required parameters were calculated as follows:

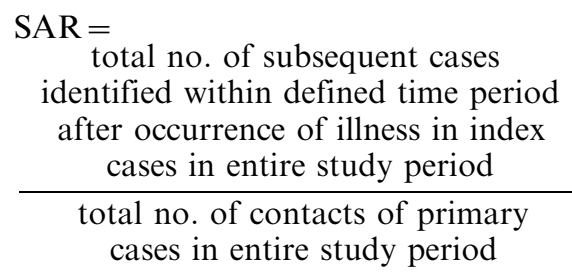

$\mathrm{ISC}=$

total no. of primary cases within

defined time period in study for

occurrence of subsequent cases population at risk

$\mathrm{RR}=\mathrm{SAR} / \mathrm{ISC}$,

$\mathrm{RD}=\mathrm{SAR}-\mathrm{ISC}$.

Taylor series $95 \%$ confidence intervals (CIs) for RR were calculated using Epi-Info v. 6.04 and CIs for $\mathrm{RD}$ were calculated based on the robust approximation of Miettinen \& Nurminen [45] using an online calculator provided by the University of Manchester (http://www.phsim.man.ac.uk/risk/). Pooled risk estimates were calculated using the METAN command in Stata version 11.0 licensed to the Robert
Koch-Institute, including an analysis of heterogeneity between studies using the $\chi^{2}$ test with a randomeffects model and weighting based on inverse variance.

\section{RESULTS}

\section{Search results}

Results of our search are summarized in Figure 1.

\section{Direct evidence}

Our search did not identify any direct evidence, i.e. studies that compared the incidence of subsequent cases in contacts given and not given chemoprophylaxis in educational settings.

\section{Indirect evidence}

A retrospective ecological study involving 12 European countries was identified in which countries with a policy of giving chemoprophylaxis only to close contacts after a single case of IMD in a preschool setting had $3 \cdot 8(95 \%$ CI $0 \cdot 7-22 \cdot 0)$ times the risk of clusters than countries with a policy of giving chemoprophylaxis to all children in the nursery [38]. There was a lack of accurate national statistics on the size and number of nursery schools. Co-primary cases were not excluded.

Our search strategy further identified seven observational studies that permitted comparison of IMD incidence in contacts of primary cases in educational settings with background incidence of sporadic IMD cases $[2-4,11,24,30,46]$ (Appendix Table 1). A total of 15 observational studies (16 publications) [2-4, 11 , $24,30,46-59]$ was identified with information on risk of subsequent IMD in household contacts of sporadic cases, of which only five contained all numerator and denominator data required for a comparison of risk in contacts who had not received chemoprophylaxis with the background incidence of sporadic cases (Appendix Table 5).

\section{Risk of subsequent cases of IMD in contacts of primary cases compared to background risk of sporadic IMD}

\section{Pre-school setting}

Five studies permitted estimation of risk in pre-school settings $[2-4,11,46]$. In two studies in which chemoprophylaxis was recommended by public health 


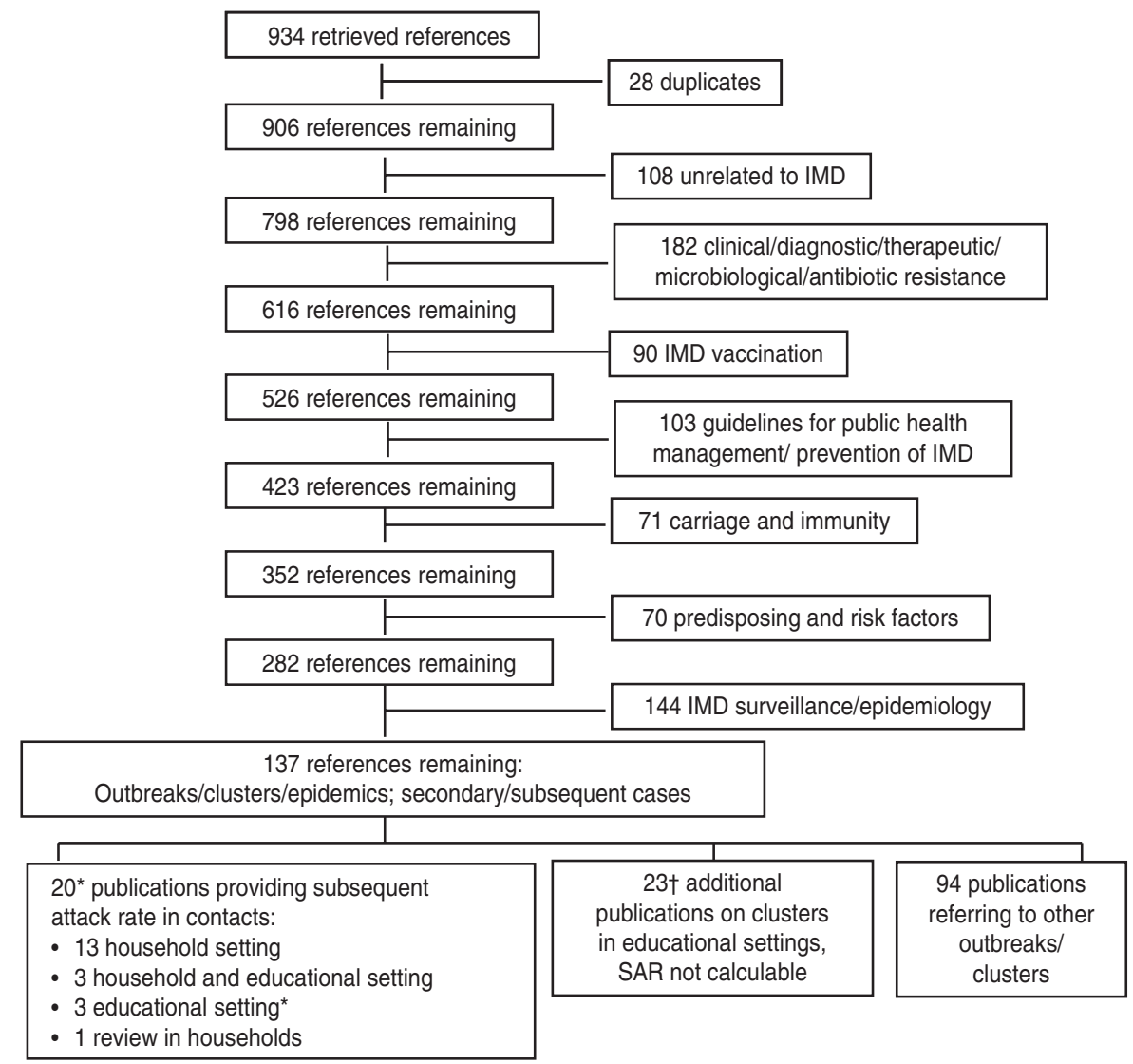

Fig. 1. Flowchart of search results. * One additional publication found in references; $\uparrow$ two additional publications found in references. IMD, Invasive meningococcal disease; SAR, subsequent attack rate.

authorities for contacts in the same pre-school as the IMD case, no subsequent cases were observed [2, 4]. In the three other studies where chemoprophylaxis was not generally recommended $[3,11,46]$, the risk of subsequent cases in contacts was significantly higher than the background IMD incidence (Appendix Table 2). The pooled estimates of RR and RD from these studies, which fulfilled criteria for homogeneity, were $22 \cdot 3(95 \%$ CI $12 \cdot 1-40 \cdot 9)$ and $58 \cdot 2 / 10^{5}(95 \%$ CI 27-3-89.0), respectively (Table 1 ).

\section{School setting}

Five studies permitted calculation of SAR, RR and $\mathrm{RD}$ in various school settings (Appendix Table 3). Chemoprophylaxis was not recommended for school contacts in these settings, with the exception of close contacts among classmates in France [4]. SAR was generally lower than in pre-school settings with the exception of the Brazilian study [26] (which was, however, undertaken in a very high incidence setting and therefore excluded from the pooled analysis) and the French study [4], where SAR estimates in classroom contacts overlapped with estimates in older preschool children in the UK [11] and Belgium [3] (Appendix Tables 2 and 3). In the one study that included both pre-school and school settings without chemoprophylaxis [11] RR and RD were markedly lower in school than nursery contacts (Appendix Table 2). The RRs were consistently statistically significantly elevated in all school-based studies, with a wide range that overlapped with RRs in pre-school settings. Because background incidences were lower in school settings, however, the RDs were consistently lower than in pre-school settings, with a pooled estimate of $4 \cdot 1 / 10^{5}(95 \%$ CI $2 \cdot 3-5 \cdot 8)$ from one US [30] and three European studies [2, 4, 11], but with significant heterogeneity between these studies (Table 1). When only data from primary-school children were pooled (possible in three studies $[2,4,11]$ ), the RD estimate was $4 \cdot 9 / 10^{5}$ (95\% CI 2.9-6.9) and heterogeneity was no longer significant. When only data from secondary-school children from these studies were pooled, heterogeneity remained significant with 
Table 1. Pooled estimates of the relative risk $(R R)$ and risk difference $(R D)$ of incidence of subsequent invasive meningococcal disease $(I M D)$ cases in contacts at $\sim 1$ month after contact with a case of IMD and background IMD incidence (detailed data in online Appendix Tables 1-5)

\begin{tabular}{|c|c|c|c|c|c|c|}
\hline Setting (references) & $\mathrm{RR}$ & $95 \% \mathrm{CI}$ & $\begin{array}{l}\text { Heterogeneity } \\
(P)\end{array}$ & $\mathrm{RD}$ & $95 \% \mathrm{CI}$ & $\begin{array}{l}\text { Heterogeneity } \\
(P)\end{array}$ \\
\hline Pre-schools $[3,11,46]$ & $22 \cdot 3$ & $12 \cdot 1$ to $40 \cdot 9$ & $0 \cdot 66$ & $58 \cdot 2$ & $27 \cdot 3$ to $89 \cdot 0$ & $0 \cdot 15$ \\
\hline Schools (all) $[2,4,11,30]$ & $26 \cdot 2$ & $12 \cdot 5$ to $54 \cdot 8$ & $<0 \cdot 0001$ & $4 \cdot 1$ & $2 \cdot 3$ to $5 \cdot 8$ & $0 \cdot 007$ \\
\hline Primary schools $[2,4,11]$ & $21 \cdot 1$ & $7 \cdot 0$ to $63 \cdot 6$ & $0 \cdot 03$ & $4 \cdot 9$ & $2 \cdot 9$ to $6 \cdot 9$ & $0 \cdot 45$ \\
\hline Secondary schools $[2,4,11]$ & $33 \cdot 3$ & $5 \cdot 1$ to $215 \cdot 7$ & $<0 \cdot 0001$ & $8 \cdot 8$ & -0.5 to $17 \cdot 7$ & $0 \cdot 004$ \\
\hline Universities [2]* & 1.5 & $0 \cdot 6$ to $3 \cdot 5$ & —* & $0 \cdot 2$ & $-0 \cdot 2$ to $0 \cdot 6$ & - \\
\hline Households $[3,4,8,56,58,60]$ & $2106 \cdot 7$ & $988 \cdot 1$ to $4491 \cdot 9$ & $0 \cdot 007$ & $418 \cdot 9$ & $282 \cdot 4$ to $555 \cdot 3$ & $0 \cdot 409$ \\
\hline \multicolumn{7}{|l|}{ Stratified: } \\
\hline$[3,4,60]$ & $1110 \cdot 2$ & $760 \cdot 1$ to $1621 \cdot 4$ & $0 \cdot 43$ & $480 \cdot 1$ & $321 \cdot 5$ to $639 \cdot 9$ & $0 \cdot 96$ \\
\hline$[8,56]$ & $6922 \cdot 1$ & $2694 \cdot 6$ to $17781 \cdot 5$ & $1 \cdot 0$ & $772 \cdot 2$ & $-1261 \cdot 8$ to $2806 \cdot 1$ & $0 \cdot 19$ \\
\hline
\end{tabular}

* One study only.

a RD estimate of $8 \cdot 8 / 10^{5}(95 \% \mathrm{CI}-0 \cdot 046$ to $17 \cdot 7)$. In school settings, RR and RD were highest when analyses were restricted to contacts in classrooms $[4,24]$ (Appendix Table 3).

\section{University setting}

Only one study [11] provided data on risk of secondary cases in the university setting (Appendix Table 4). The size of the contact group was very large $(>5000)$ and the SAR was only marginally higher than baseline (RR 1.5, $95 \%$ CI 0.6-3.5).

\section{Household setting}

For comparison with the educational settings, in the five household studies (Appendix Table 5) the pooled RRs and RDs were 2254 (95\% CI 947-5362) and $401 / 10^{5}$ (95\% CI 263-539), respectively (Table 1 ). The observed substantial heterogeneity in the RR estimate from these five studies was resolved by stratification, leading to a more conservative estimate of RR of $1110 \cdot 2(95 \%$ CI $760 \cdot 1-1621 \cdot 4)$ but a slightly higher RD of $480 \cdot 1(95 \%$ CI 321.5-639.9) when the two studies with extremely high RR (and low number of primary and secondary cases) $[8,56]$ were excluded (Table 1).

\section{Timing and exact setting of subsequent cases within educational institutions}

Exact data on the time interval between occurrence of the primary and subsequent cases were not available in all studies. Available data in two studies suggested that about $70 \%$ of subsequent cases occurred within
1 week and 90-100\% within 3 weeks $[3,11]$. In the US study in schools, $33 \%$ of subsequent cases occurred within 1-2 days and $73 \%$ within 14 days [30]. Davison et al. [11] reported that $57 \%$ of all subsequent cases occurred in the same grade or class in pre-school and school settings combined, and Zangwill et al. [30] reported that $55 \%$ of subsequent cases were in a different grade than the index case.

\section{DISCUSSION}

Our search did not identify direct evidence for the effectiveness of chemoprophylaxis in contacts of IMD in educational settings as no studies compared the incidence of subsequent cases in treated and untreated contacts. However, we found indirect evidence that permitted a comparison of the risk of subsequent cases in contacts of persons with IMD in educational settings with the background risk in a defined time interval of $\sim 30$ days after occurrence of the index case. Because direct evidence exists for the effectiveness of chemoprophylaxis of contacts in household settings, we also calculated the risk of subsequent cases in household settings in contacts who did not receive chemoprophylaxis and compared this to background risk. Our study showed that contacts in pre-school and school settings in which chemoprophylaxis was not generally recommended had a significantly increased risk of IMD, but that this risk was markedly lower than for household contacts.

The studies included in our analysis have a number of limitations. While they applied directly to the populations of interest, background incidences varied. 
Active prospective follow-up of contacts to ascertain subsequent cases was not performed in all household studies (Appendix Table 1). All key studies in educational settings but one collected at least some of the data retrospectively (Appendix Table 1) and lacked data on potential confounding variables such as socioeconomic factors and other risk factors for IMD. Some studies included co-primary cases [2,11], which would lead to an overestimation of SAR and RR. In addition, the studies varied in the definition of the time interval for ascertainment of subsequent cases, which ranged from 28 days to 4 months. However, as the risk of subsequent cases approaches the background risk after 3-4 weeks, the RR and RD would tend to be lower by inclusion of studies with a longer observation period. In all studies, the number of contacts of primary cases was estimated based on available national data on mean group or class size and size of institutions and thus probably diverged from the true situation in the few outbreaks that occurred. Furthermore, calculation of SAR was often based on very small numbers of subsequent cases (sparse data), and the observed increased risk of IMD in contacts vs. the background risk tended to be higher in less precise studies with smaller numbers of cases. Moreover, recognition of a case among contacts may be more likely than in the general population, thus background incidence may be underestimated and RR overestimated. However, as IMD is such a severe disease, under-ascertainment of primary cases is likely to be lower than for many other infections. Data were lacking on whether any contacts actually obtained chemoprophylaxis; information was only given as to whether chemoprophylaxis was generally recommended or not in the respective setting when the study was undertaken. Receipt of antibiotics by contacts would lead to falsely low estimates of SAR. Only three studies $[2,11,30]$ provided data on strain characterization of at least a proportion of primary and subsequent cases; these suggested that meningococcal strains are identical in primary and subsequent cases in most instances.

In view of these limitations it is, nonetheless, remarkable that the evaluated studies consistently showed a significantly increased risk for the occurrence of subsequent IMD cases compared to the background incidence of primary cases in a time interval from $0-2$ to $28-120$ days after illness onset in the index case in pre-school and school settings where chemoprophylaxis was not generally recommended. Compared to background incidence, the risk of acquiring IMD was significantly elevated in preschool settings (RD $58 \cdot 2 / 10^{5}$, 95\% CI $27 \cdot 3-89 \cdot 0$ ) when chemoprophylaxis was not recommended. This is underscored by the absence of subsequent cases in pre-school contacts in settings with clear recommendations for chemoprophylaxis in place $[2,4]$. In fact, in England and Wales, no subsequent cases of IMD were observed in pre-school settings from 1992 to 1995 , when chemoprophylaxis was recommended, but were observed starting in 1995-1996, when this recommendation was rescinded. While clusters due to serogroup C did not occur after 1999-2000, when mass vaccination against IMD due to serogroup $C$ took place in the UK [61], serogroup B clusters continued to be observed in educational settings [12, 62]. Our findings are also supported by an analysis of IMD clusters in Barcelona from May 1995 to December 1997 [5], in which seven of the 13 observed clusters occurred in nurseries [two occurred in schools, one in siblings (family members received chemoprophylaxis in $93.6 \%$ of cases), two in military settings and one in members of the same village]. Our estimated RD for pre-schools was an order of magnitude lower than that for household settings $\left(401 / 10^{5}, 95 \%\right.$ CI 263-539), where chemoprophylaxis has been estimated to decrease the risk of subsequent cases in contacts by $\sim 86 \%$ [44]. Under the assumption that chemoprophylaxis would be similarly effective in preschool settings, the number of contacts to be treated (NNT) with an antibiotic to prevent one case can be estimated as RD $1 / 0 \cdot 86$. Based on the above data, this yields an estimate of 1998 (95\% CI 1307-4259) children, compared to 290 (95\% CI 216-442) in household settings. Thus the cost of preventing one case would be markedly higher than in households. Furthermore, in educational settings it may take longer or be more complex to arrange for all contacts to receive antibiotics, thus the effectiveness of chemoprophylaxis may well be lower than in households.

While RR estimates overlapped, RD estimates in school settings were an order of magnitude lower than in pre-school settings, largely because the background risk in older children was lower than in younger children. Thus, NNT estimates would be markedly higher still. The study in university settings was limited by the large size of the contact group, which may have made recognition of epidemiologically linked cases more difficult; defining smaller contact groups might have led to higher estimates of SAR.

None of the studies included in the meta-analyses mentioned educational workers. A review article on 
the risk of educational workers [63] listed only one reference that identified teachers who contracted IMD. In this study, which reviewed 50 adult IMD cases ascertained from 1997 to 1999 in Cheshire, UK, seven cases were identified in educational workers with a sixfold higher risk compared to the general adult working population [64]. The authors recommended caution in interpretation of these results due to the small area investigated during a period of increased incidence; however, the results suggest that school workers should be included in the risk assessment of contacts of IMD cases.

While a review of 18 studies did not find any reports of severe adverse events in conjunction with antimicrobial chemoprophylaxis with rifampicin, cipropfloxacin, ceftriaxone or azithromyicin, mild sideeffects of recommended antibiotic regimens were common [65]. A case of anaphylaxis and two anaphylactoid reactions have been described in conjunction with chemoprophylaxis using ciprofloxacin [65-67]. Thus severe side-effects may rarely occur, particularly if the number of persons defined as contacts is large. The development of resistance has been observed in randomized controlled eradication trials with rifampicin, with $10-27 \%$ of initial carriers developing resistance in three randomized controlled trials [68-71]. Emergence and spread of rifampicin resistance has also been observed in several non-controlled studies $[22,48,72,73]$ and cases due to rifampicin-resistant meningococcal isolates have also been reported after prophylaxis [48, 74-77]. On the other hand, resistant $N$. meningitidis has not spread widely, possibly because the acquisition of rifampicin resistance appears to confer a biological disadvantage [71]. Ciprofloxacin-resistant $N$. meningitidis was isolated in the USA in 2009 from three IMD cases and two carriers, one after contact with one of the patients and after receipt of ciprofloxacin [78]; no further cases have been described after recommendations to use other antibiotics for prophylaxis in the affected regions. Another theoretical negative effect of chemoprophylaxis is the eradication of $N$. lactamica from the nasopharynx. Colonization of $N$. lactamica is associated with the induction of cross-protective immunity to $N$. meningitides $[79,80]$. Carriage of $N$. lactamica is highest in nursery-aged children $[79,81]$ and prior antibiotic therapy has been shown to decrease carriage [81].

Because IMD is associated with a high risk of complications and death its occurrence generates substantial anxiety in contacts [82]. Thus we believe that contacts would want chemoprophylaxis even if evidence for benefit is weak, as direct harmful effects are rare and further risks largely theoretical. This is in keeping with comments from The Meningitis Trust, a non-governmental organization in the UK with a public helpline, that it is difficult to convince parents of children attending the same nursery/playgroup as a case that prophylaxis is not needed.

As described in detail elsewhere [44], GRADE methodology was applied to the results of this review after taking into account the above limitations and after weighing the potential benefits and harms in light of disease severity and risk in the settings. This resulted in the recommendation that attending the same pre-school as an IMD case should be considered an indication for chemoprophylaxis, depending on risk assessment. The risk assessment should take into account duration and closeness of contact, as the risk of further cases is likely to be higher in settings similar to households, where risk of exposure to respiratory droplets would be more likely. Some studies found a higher risk for IMD in more crowded household settings [51, 83-85] and crowded conditions were described in several day-care-associated outbreaks in the USA $[13,14]$. Thus children in the same group as the index case who have spent long periods in the same room (e.g. full-time attendance, sharing meals, napping together) are likely to be at higher risk than children in a different group.

The results of this systematic review suggest that attending the same school/college (including the same class) as a first case of IMD should not in itself be considered an indication for widespread chemoprophylaxis in contacts. Here also, the decision for whom chemoprophylaxis is indicated should be based on an assessment of whether household-like contact may have occurred among school friends, e.g. spending nights together. In two school outbreaks various extracurricular activities or excursions were identified as possible settings in which transmission may have occurred [25, 27].

Regardless of whether they receive chemoprophylaxis, all contacts should be informed of early symptoms of disease and the importance of seeking immediate medical advice, as failure to do so has been described and re-introduction of the index strain cannot be ruled out due to the large number of social contacts involved in educational settings. Indirect evidence is available suggesting that vaccination of close contacts of index cases with IMD due to a vaccine-preventable strain prevents late secondary 
cases [86], and thus post-exposure vaccination is strongly recommended in the ECDC guidance document [44]. Further prospective studies on the risk of subsequent cases and the transmission of diseasecausing strains in educational settings are needed. Prospective studies on the risk of subsequent cases in contacts who receive and do not receive chemoprophylaxis may be feasible as an initiative involving several countries with divergent public health policies.

In conclusion, we found limited but consistent evidence that the risk of IMD in pre-school contacts of sporadic cases is significantly increased above the background risk in European settings. In addition, in two studies performed in settings with a recommendation for prophylactic treatment of preschool contacts, no subsequent cases were observed. This is in keeping with results of the retrospective ecological study performed by Boccia et al. [38], which suggested a lower risk of IMD clusters in countries in which prophylaxis of pre-school contacts was recommended compared to those without such a recommendation, although the difference was not statistically significant. Although direct evidence for the effectiveness of chemoprophylaxis has only been shown in household settings, where the risk of subsequent cases is an order of magnitude higher, we recommend chemoprophylaxis for pre-school contacts based on an assessment of duration and closeness of contact. In school or college settings chemoprophylaxis should be offered only when there is evidence of close prolonged contact with the index case.

\section{NOTE}

Supplementary material accompanies this paper on the Journal's website (http://journals.cambridge. org/hyg).

\section{ACKNOWLEDGEMENTS}

This study was funded in part by the European Center for Disease Prevention and Control (ECDC). We thank Pierluigi Lopalco and Helena de Carvalho Gomes from ECDC for technical and expert advice. We thank Ole Wichmann for helpful comments on the manuscript.

\section{DECLARATION OF INTEREST}

None.

\section{REFERENCES}

1. Trotter CL, Gay NJ, Edmunds WJ. The natural history of meningococcal carriage and disease. Epidemiology and Infection 2006; 134: 556-566.

2. Hastings $\mathbf{L}$, et al. A retrospective survey of clusters of meningococcal disease in England and Wales, 1993 to 1995: estimated risks of further cases in household and educational settings. Communicable Disease Report 1997; 7: R195-R200.

3. De Wals P, et al. Meningococcal disease in Belgium. Secondary attack rate among household, day-care nursery and pre-elementary school contacts. Journal of Infection 1981 ; 3: 53-61.

4. Olivares R, Hubert B. Clusters of meningococcal disease in France (1987-1988). European Journal of Epidemiology 1992; 8: 737-742.

5. Palau AO, Noguera HP. Factors predictive of secondary cases of meningococcal disease in Barcelona. Epidemiology of the disease [in Spanish]. Revista española de salud pública 1998; 72: 443-450.

6. Samuelsson S, et al. An outbreak of serogroup B:15:P1.16 meningococcal disease, Frederiksborg County, Denmark, 1987-9. Epidemiology and Infection 1992; 108: 19-30.

7. Purcell B, et al. Effectiveness of antibiotics in preventing meningococcal disease after a case: systematic review. British Medical Journal 2004; 328: 1339 1340 .

8. Stefanoff P, et al. The detection of meningococcal household clusters and their prophylaxis in the changing epidemiological situation of invasive meningococcal disease in poland, 2003-2006. Eurosurveillance 2008; 13: 44-46.

9. Bernabeu MF, et al. Incidence of secondary meningococcal outbreak among school children younger than five years [in Spanish]. Medicina Clinica 1980; 74: 142-144.

10. Cartwright KA, Hunt D, Fox A. Chemoprophylaxis fails to prevent a second case of meningococcal disease in a day nursery. Communicable Disease Report CDR Review 1995; 5: R199.

11. Davison KL, et al. Clusters of meningococcal disease in school and preschool settings in England and Wales: what is the risk? Archives of Disease in Childhood 2004; 89: 256-260.

12. Granerod J, et al. Clusters of meningococcal disease in educational establishments in the United Kingdom: April 2001 to March 2002. Communicable Disease and Public Health/PHLS 2004; 7: 51-55.

13. Jacobson JA, Filice GA, Holloway JT. Meningococcal disease in day-care centers. Pediatrics 1977; 59: 299-300.

14. Leggiadro RJ, et al. Invasive meningococcal disease: secondary spread in a day-care center. Southern Medical Journal 2000; 82: 511-513.

15. O'Donovan D, et al. Outbreak of group C meningococcal infection affecting two preschool nurseries. Communicable Disease and Public Health/PHLS 2000; 3: $177-180$. 
16. Sáez-Nieto JA, et al. Outbreak of infection caused by Neisseria meningitidis group $\mathrm{C}$ type 2 in a nursery. Journal of Infection 1984; 8: 49-55.

17. Samuelsson S, Gustavsen S, Rønne T. Epidemiology of meningococcal disease in Denmark 1980-1988. Scandinavian Journal of Infectious Diseases 1991; 23: 723-730.

18. Davison RP, et al. Using the national guidelines to manage a meningococcal group $\mathrm{C}$ outbreak in a Brisbane boarding school-some discretionary judgements are needed. Communicable Diseases Intelligence 2003; 27: 520-523.

19. Feigin RD, et al. Epidemic meningococcal disease in an elementary school classroom. New England Journal of Medicine 1982; 307: 1255-1257.

20. González de Aledo LA, García MJ. Control of a school outbreak of serogroup B meningococcal disease by chemoprophylaxis with azithromycin and ciprofloxacin [in Spanish]. Anales españoles de pediatría 2000; 53: 412-417.

21. Hudson PJ, et al. Evidence for school transmission of Neisseria meningitidis during a Vermont outbreak. Pediatric Infectious Disease Journal 1986; 5: 213-217.

22. Jackson LA, et al. Evaluation of the use of mass chemoprophylaxis during a school outbreak of enzyme type 5 serogroup B meningococcal disease. Pediatric Infectious Disease Journal 1996; 15: 992-998.

23. Jackson LA, et al. Serogroup C meningococcal outbreaks in the United States. An emerging threat. Journal of the American Medical Association 1995; 273: 383-389.

24. Jacobson JA, et al. The risk of meningitis among classroom contacts during an epidemic of meningococcal disease. American Journal of Epidemiology 1976; 104: 552-525.

25. Morrow HWM, et al. Risk factors associated with a school-related outbreak of serogroup C meningococcal disease. Pediatric Infectious Disease Journal 1990; 9: 394-397.

26. Oppermann H, et al. Meningococcal carriage in high school students and possible risk factors [in German]. Das Gesundheitswesen 2006; 68: 633-637.

27. Robinson P, et al. An outbreak of serogroup C meningococcal disease associated with a secondary school. Communicable Diseases Intelligence 2001; 25: 121-125.

28. Schubiger G, et al. Meningococcal epidemic in a boarding school: a rifampicin-resistant secondary case while under chemoprophylaxis [in German]. Schweizerische Medizinische Wochenschrift 1986; 116: 1172-1175.

29. Sutton T, Ip S. A cluster of meningococcal meningitis cases in an Auckland secondary school. New Zealand Medical Journal 1987; 100: 153.

30. Zangwill KM, et al. School-based clusters of meningococcal disease in the United States. Descriptive epidemiology and a case-control analysis. Journal of the American Medical Association 1997; 277: 389-395.

31. Barker RM, et al. Practical guidelines for responding to an outbreak of meningococcal disease among university students based on experience in Southampton. Communicable Disease and Public Health/PHLS 1999; 2: 168-173.

32. Ferson M, et al. Unusual cluster of mild invasive serogroup $\mathrm{C}$ meningococcal infection in a university college. Communicable Diseases Intelligence 1999; 23: 261-264.

33. Riordan T. A college outbreak of group C meningococcal infection: how widely should investigation and prophylaxis extend. Communicable Disease Report. CDR Review 1997; 7: 5-9.

34. Round A, et al. Public health management of an outbreak of group $\mathrm{C}$ meningococcal disease in university campus residents. European Journal of Public Health 2001; 11: 431-436.

35. Bruce MG, et al. Risk factors for meningococcal disease in college students. Journal of the American Medical Association 2001; 286: 688-693.

36. Kristiansen BE, Tveten Y, Jenkins A. Which contacts of patients with meningococcal disease carry the pathogenic strain of Neisseria meningitidis? A population based study. British Medical Journal 1998; 317: 621-625.

37. Wall R, et al. Meningococcal infection: evidence for school transmission. Journal of Infection 1991; 23: $155-159$.

38. Boccia D, et al. Effectiveness of different policies in preventing meningococcal disease clusters following a single case in day-care and pre-school settings in Europe. Epidemiology and Infection 2006; 134: 872-877.

39. Hoek M, et al. A European survey on public health policies for managing cases of meningococcal disease and their contacts. Eurosurveillance 2008; 13: 78-80.

40. Atkins D, et al. Systems for grading the quality of evidence and the strength of recommendations I: Critical appraisal of existing approaches The GRADE Working Group. BMC Health Services Research 2004; 4: 38.

41. Cochrane Collaboration. Cochrane Handbook for Systematic Reviews of Interventions, version 5.0.2 (updated September 2009), 2009.

42. Schünemann HJ, et al. Transparent development of the WHO Rapid Advice Guidelines. PLoS Medicine 2007; 4: e119.

43. Schünemann S, et al. WHO Rapid Advice Guidelines for pharmacological management of sporadic human infection with avian influenza A (H5N1) virus. Lancet Infectious Diseases 2007; 7: 21-31.

44. European Centre for Disease Prevention and Control. Public health management of sporadic cases of invasive meningococcal disease and their contacts. Stockholm, 2010.

45. Miettinen OS, Nurminen M. Comparative analysis of two rates. Statistics in Medicine 1985; 4: 213-226.

46. Favorova LA, Sokova IN, Chernyshova TF. Results of controlled epidemiological trial on the use of placental gamma globulin in foci of meningococcal infection. Zhurnal Mikrobiologii, Epidemiologii i Immunobiologii $1975 ; 6: 15-18$. 
47. Ackerman JH. Meningococcal disease in Ohio. Ohio State Medical Journal 2010; 70: 710-712.

48. Cooke RP, et al. Secondary cases of meningococcal infection among close family and household contacts in England and Wales, 1984-7. British Medical Journal 1989; 298: 555-558.

49. Hoiby EA, et al. 23. Phenoxymethyl-penicillin treatment of household contacts of meningococcal disease patients. Antonie van Leeuwenhoek, International Journal of General and Molecular Microbiology 1986; 52: 255-257.

50. Juels C, et al. Meningococcal disease in California. Epidemiology and management. Western Journal of Medicine 1978; 128: 195-202.

51. Kaiser AB, et al. Seroepidemiology and chemoprophylaxis disease due to sulfonamide-resistant Neisseria meningitidis in a civilian population. Journal of Infectious Diseases 1974; 130: 217-224.

52. Marks MI, Frasch CE, Shapera RM. Meningococcal colonization and infection in children and their household contacts. American Journal of Epidemiology 1979; 109: 563-571.

53. Munford RS, et al. Spread of meningococcal infection within households. Lancet 1974; 303: 1275-1278.

54. Norton JF. Meningococcus meningitis in Detroit: 1928-1929. Journal of Preventive Medicine 1931; 5: 365-367.

55. Olcen P, et al. Epidemiology of Neisseria meningitidis; prevalence and symptoms from the upper respiratory tract in family members to patients with meningococcal disease. Scandinavian Journal of Infectious Diseases 1981; 13: 105-109.

56. Samuelsson S, et al. Prevention of secondary cases of meningococcal disease in Denmark. Epidemiology and Infection 2000; 124: 433-440.

57. Scholten RJPM, et al. Upper respiratory tract infection, heterologous immunisation and meningococcal disease. Journal of Medical Microbiology 2009; 48: 943-946.

58. The Meningococcal Disease Surveillance Group. Meningococcal disease. Secondary attack rate and chemoprophylaxis in the United States, 1974. Journal of the American Medical Association 1974; 235: 261-265.

59. The Meningococcal Disease Surveillance Group. Analysis of endemic meningococcal disease by serogroup and evaluation of chemoprophylaxis. Journal of Infectious Diseases 1976; 134: 201-204.

60. Scholten RJ, et al. Secondary cases of meningococcal disease in the Netherlands, 1989-1990, a reappraisal of chemoprophylaxis [in Dutch]. Nederlands Tijdschrift voor Geneeskunde 1993; 137: 1505-1508.

61. Trotter CL, Ramsay ME. Vaccination against meningococcal disease in Europe: review and recommendations for the use of conjugate vaccines. FEMS Microbiology Reviews 2007; 31: 101-107.

62. Health Protection Agency. Clusters of meningococcal disease in educational institutions in England, Wales and Northern Ireland, April 1995-March 2003 (http://www.hpa.org.uk/Topics/InfectiousDiseases/ InfectionsAZ/MeningococcalDisease/ClustersIn EducationalInstitutions/menin_ClusterUpdateTables/).
63. De Wals $\mathbf{P}$, et al. Risk and prevention of meningococcal disease among educations workers: A review. Canadian Journal of Infectious Diseases 2004; 15: 89-93.

64. Woodhouse S, Hunter PR. Risk of invasive meningococcal disease among school workers in Cheshire, United Kingdom. Clinical Infectious Diseases 2001; 32: 1795-1797.

65. Fraser A, et al. Antibiotics for preventing meningococcal infections [Review]. The Cochrane Library 2005, pp. 1-35.

66. Burke P, Burne SR. Allergy associated with ciprofloxacin. British Medical Journal 2000; 320: 679.

67. Giovanetti F. Anaphylaxis following unnecessary meningococcal chemoprophylaxis of a healthcare worker. Eurosurveillance 2009; 14: 351-353.

68. Blakebrough IS, Gilles HM. The effect of rifampicin on meningococcal carriage in family contacts in northern Nigeria. Journal of Infection 1980; 2: 137-143.

69. Guttler RB, et al. Rifampin and minocycline on meningococcal carrier rates. Journal of Infectious Diseases 1971; 124: 199-205.

70. Munford RS, et al. Eradication of carriage of Neisseria meningitidis in families: a Study in Brazil. Journal of Infectious Diseases 1974; 129: 644-649.

71. Taha M-K, et al. Rifampin-resistant Neisseria meningitidis. Emerging Infectious Diseases 2006; 12: 859-860.

72. Devine LF, et al. Field trial of the efficacy of a previously proposed regimen using minocycline and rifampin sequentially for the elimination of meningococci from healthy carriers. American Journal of Epidemiology 1973; 97: 394-401.

73. Lepe JA, et al. Evolution of Neisseria meningitidis sensitivity to various antimicrobial drugs over the course of chemoprophylaxis during an epidemic outbreak. Enfermedades Infecciosas y Microbiologia Clinica 2006; 24: 608-612.

74. Almog R, et al. First recorded outbreaks of meningococcal disease in the Israel Defence Force: three clusters due to serogroup $\mathrm{C}$ and the emergence of resistance to rifampicin. Infection 1994; 22: 69-71.

75. Cooper ER, et al. Rifampin-resistant meningococcal disease in a contact patient given prophylactic rifampin. Journal of Pediatrics 1986; 108: 93-96.

76. Dawson SJ, Fey RE, McNulty CA. Meningococcal disease in siblings caused by rifampicin sensitive and rifampicin resistant strains. Communicable disease and public health/PHLS 1999; 2: 215-6.

77. Rainbow J, et al. Rifampin-resistant meningococcal disease. Journal of Emerging Infectious Diseases 2005; 11: 977-979.

78. Wu HM, et al. Emergence of ciprofloxacin-resistant Neisseria meningitidis in North America. New England Journal of Medicine 2009; 360: 886-892.

79. Gold R, et al. Carriage of Neisseria meningitidis and Neisseria lactamica in infants and children. Journal of Infectious Diseases 1978; 137: 112-21.

80. Coen PG, Cartwright K, Stuart J. Mathematical modelling of infection and disease due to Neisseria meningitidis and Neisseria lactamica. International Journal of Epidemiology 2000; 29: 180-188. 
81. Bakir M, et al. Asymptomatic carriage of Neisseria meningitidis and Neisseria lactamica in relation to Streptococcus pneumoniae and Haemophilus influenzae colonization in healthy children: apropos of 1400 children sampled. European Journal of Epidemiology 2002; 17: 1015-1018.

82. Taylor-Robinson D, et al. Students' attitudes to the communications employed during an outbreak of meningococcal disease in a UK school: a qualitative study. Journal of Public Health 2010; 32: 32-37.

83. Baker M, et al. Household crowding a major risk factor for epidemic meningococcal disease in Auckland children. Pediatric Infectious Disease Journal 2000; 19: 983-990.

84. Grein T, Flanagan D. Day-care and meningococcal disease in young children. Epidemiology and Infection 2001; 127: 435-441.

85. McCall BJ, Neill AS, Young MM. Risk factors for invasive meningococcal disease in southern Queensland, 2000-2001. Internal Medicine Journal 2004; 34 : 464-468.

86. Hoek M, et al. Effectiveness of vaccinating household contacts in addition to chemoprophylaxis after a case of meningococcal disease: a systematic review. Epidemiology and Infection 2008; 136: 1441-1447. 